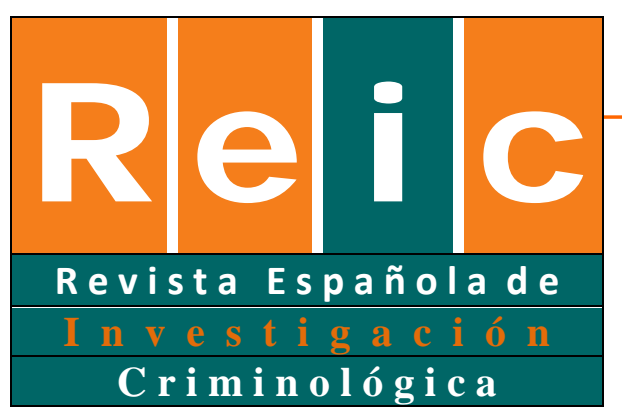

Igareda \& Bodelón

\title{
Las violencias sexuales en las universidades: cuando lo que no se denuncia no existe.
}

\section{Sexual violences at university: when what it is not denounced it does not exist.}

Manuscrito recibido 5 diciembre 2013/Publicado el 19 de marzo de 2014

\author{
Noelia Igareda y Encarna Bodelón ${ }^{1}$ \\ Universidad Autónoma de Barcelona
}

"Las narraciones que examinamos en este libro fueron cruciales para crear al sujeto sexual; pero ninguna persona está implacablemente encerrada en el marco de estos guiones abusivos. Estas personas realizan sus elecciones de entre un fondo de significados en circulación. Los violadores no nacen; se hacen (...) Podemos forjar un futuro sin violencia sexual.” (Bourke, 2009:27).

\section{RESUMEN}

Las violencias sexuales tienen lugar en las universidades, como en cualquier otro entorno de nuestra sociedad. La violencia sexual, como una forma de violencia de género, cruza clases sociales, niveles educativos, culturas y religiones. Se tiende a pensar que el mayor nivel educacional de las estudiantes es una herramienta suficiente de prevención y actuación frente a una eventual agresión sexual. El presente artículo muestra a través de una investigación cualitativa en universidades españolas la experiencia de las estudiantes frente a la violencia sexual, para entender por qué no lo comunican y/o denuncian. La falta de denuncias conduce a las autoridades universitarias a considerar que se trata de un fenómeno muy extraño en los campus universitarios. De ahí, la invisibilidad del fenómeno, y también la falta de medidas de actuación específica de prevención y actuación en casos de violencia de género, y violencia sexual en particular.

\footnotetext{
${ }^{1}$ La correspondencia debe enviarse a Noelia Igareda, Departamento de Ciencia Política y Derecho Público, Facultad de Derecho, Edifici B, campus UAB, 08193 Bellaterra (Cerdanyola del Vallés), Barcelona, Spain. Noelia.igareda@uab.cat
} 
Palabras clave: violencia sexual, universidad, estudiantes, falta de denuncia.

\begin{abstract}
Sexual violence takes place within universities, as in any other place in society. Sexual violence, as a form of gender violence, crosses all social classes, educational levels, cultures and religions. It is generally understood that a higher level of education of female university students is a sufficient tool of prevention and answer in front of an eventual sexual aggression. The present article shows through a qualitative research done at Spanish universities the female students' experiences on sexual violence, to understand why they do not inform and/or denounce. The lack of denounces, makes the university authorities believe that it is a very rare phenomenon within the university campuses. This explains in part the invisibility of the problem, and also, the lack of specific measures of prevention and response in cases of gender violence, and sexual violence in particular.
\end{abstract}

Key words: sexual violence, university, female students, lack of denounce.

\title{
1. Introducción
}

Las violencias sexuales ${ }^{2}$ contra las mujeres son tal vez una de las formas de violencia de género más graves, ya que el ataque sexual constituye una de las formas de violación de los derechos humanos de las mujeres que produce consecuencias más devastadoras en la vida, dignidad y calidad de vida de éstas. Este tipo de conductas no son un ataque infrecuente, sino que las mujeres a lo largo de su vida tienen un alta probabilidad de sufrir estos tipos de violencias de género. Así, la Enquesta de violència masclista a Catalunya, realizada en el $2010^{3}$ indica que a lo largo de su vida un 6,2\% de las mujeres han sufrido intentos de violación, un 2,9\% una violación.

\footnotetext{
${ }^{2}$ En este artículo se entiende por "violencias sexuales", no sólo las agresiones sexuales, sino también las conductas de acoso sexual y por razón de sexo y de stalking.

${ }^{3}$ Los resultados de esta encuesta han sido publicados parcialmente a través de un resumen que se puede obtener en Generalitat de Catalunya (2010). La metodología completa de la encuesta no ha sido publicada. Con relación al tema de agresiones sexuales, no se indica si para obtener los resultados sobre la conducta de agresiones sexuales se ha utilizado una expresión poco clara y que puede comprender muchas conductas como la expresión "violación” o si se ha seguido la estrategia metodológica más correcta que sería efectuar la pregunta describiendo detalladamente diversas conductas de agresión sexual. Esta es la estrategia que utilizan las encuestas más modernas como y las que recomiendan algunas guías sobre el tema como la realizada por la Organización Mundial de la Salud (WHO, 2005).
} 
En la sociedad está muy arraigada la creencia de que las violencias de género suceden en mayor medida entre los grupos de población con menores niveles económicos, educativos y sociales. A pesar de ello, numerosas investigaciones han demostrado que la violencia de género atraviesa todas las clases sociales, culturas y niveles educativos (Dobash y Dobash, 1992; Cantera, 1999; Lorente, 2001; Gil, 2007).

No obstante, la universidad se percibe por parte de la propia comunidad universitaria como un lugar libre de este tipo de violencias, y especialmente de cualquier forma de violencia sexual, porque tanto los hombres como las mujeres que participan en ella (sean estudiantes, profesorado o personal no académico) se presupone que su mayor nivel educativo y formación intelectual les convierte a los primeros en imposibles agresores, y a las segundas en personas con suficientes herramientas intelectuales como para prevenir, y defenderse, en su caso frente a una eventual agresión, a pesar de que las investigaciones existentes en la universidad en el mundo anglosajón y en Europa muestren que la universidad no está ajena a este fenómeno (Valls et al. 2007).

Sin embargo, las encuestas de victimización nos muestras que las mujeres con estudios universitarios no están libres de sufrir violencias machistas. En el caso de L'enquesta de violència masclista a Catalunya (Generalitat de Catalunya, 2010), la prevalencia de la violencia machista de especial impacto subjetivo, como es el caso de las agresiones sexuales, en función del nivel educativo aporta los siguientes datos: un $7,7 \%$ entre las que tienen estudios primarios, un 10,6\% entra las que tienen secundarios obligatorios, un 9,9\% entre las que tienen estudios secundarios post-obligatorios, y un 8,8\% entre las que tienen estudios superiores ${ }^{4}$. Es decir, en el conjunto de la población femenina las mujeres universitarias tendrían una cifra de victimización muy semejante a otros colectivos e incluso superior al colectivo con estudios primarios. Ahora bien, la misma encuesta indica que comparando franjas de edad, las mujeres entre 18 y 29 años y con estudios primarios son objeto de una victimización del 20,9\%, mientras que la prevalencia entre mujeres con estudios superiores de esa misma franja de edad sería del 9,5\%. Eso indicaría una victimización menor de las mujeres universitarias jóvenes que la de otras mujeres jóvenes. Sin embargo, el dato de un 9.5\% de agresiones de especial 
impacto entre mujeres jóvenes universitarias resulta muy preocupante y niega la presunta ausencia de violencia entre la población universitaria femenina.

Otros datos que indican la importancia de las violencias machistas entre las mujeres universitarias son los resultados de la reciente encuesta europea sobre violencia de género en la Unión Europea, en la que se afirma: "Entre las mujeres con solo educación primaria, el 23\% han tenido experiencias de violencia física y/o sexual por parte de una pareja desde la edad de 15 años, el 21\% de las mujeres con educación secundaria y el 21\% de las mujeres con educación superior (...) Sin embargo, las mujeres con educación superior experimentan los niveles más altos de violencia por parte de sus parejas, dado que: el 19\% de las mujeres con educación primaria han sufrido violencia física y/o sexual por parte de persones que no eran sus parejas desde la edad de 15 años, mientras que esa misma situación se da en un 22\% de las mujeres con educación secundaria y en un 27\% de las mujeres con educación superior...” (European Union Agency for Fundamental Rights, 2014: 36).

Frente a esto, los esfuerzos de las autoridades universitarias en materia de seguridad y prevención del delito se centran en todo caso, en cuestiones ligadas a los ataques a la propiedad privada, y a posibles atraques contra las personas cometidas por personas ajenas a la comunidad universitaria, y vinculando todo ello, a políticas sobre la seguridad en el espacio público, la construcción de los edificios y otros espacios universitarios (Hanmer et al. 1989; Stanko, 1990).

El presente artículo pretende exponer algunas conclusiones extraídas en una investigación llevada a cabo en el marco de un proyecto europeo sobre violencia sexual en las universidades. En el caso español, se recogió información sobre la incidencia de la violencia sexual en la población universitaria femenina en cinco universidades del Estado, y sobre las respuestas institucionales a este tipo de incidentes en una de ellas.

La hipótesis general de esta investigación es que a pesar de la ausencia de denuncias de violencia sexual en la universidad, dicho fenómeno también afecta de forma grave a las estudiantes.

El objetivo de esta investigación fue verificar que la violencia de género, en sus diversas manifestaciones, también afecta a las mujeres de la comunidad universitaria. Dado que dichas conductas no son en general denunciadas se diseñó una metodología cuantitativa (cuestionario on-line anónimo a la población universitaria femenina) y 
cualitativa (grupos de discusión con estudiantes femeninos de grado, postgrado, máster y doctorado) que permitiera visibilizar la realidad del fenómeno y sus características en el contexto universitario.

De toda esta investigación, el presente artículo se centra en mostrar la experiencia de las estudiantes universitarias frente a la violencia sexual en las universidades, para así poder entender por qué no lo comunican y/o denuncian, y en consecuencia, por qué las autoridades universitarias desconocen el fenómeno y rara vez actúan. Por este motivo, el artículo se centrará en los resultados cualitativos del proyecto $^{5}$.

\section{Metodología: la experiencia de las estudiantes y la opinión de los agentes}

La presente investigación tenía dos dimensiones metodológicas: el trabajo de campo cualitativo $^{6}$, que tuvo lugar en la Universidad Autónoma de Barcelona (UAB) y las encuestas on-line, que se llevaron a cabo en la UAB, en la Universitat de Girona, la Universidad de Lleida, la Universidad de Zaragoza y la Universidad de Huelva ${ }^{7}$.

La metodología cualitativa se escogió para conocer la experiencia de las estudiantes universitarias sobre el fenómeno de la violencia sexual: su identificación,

\footnotetext{
${ }^{5}$ Los resultados cuantitativos han sido publicados en en parte en Igareda, N. y Bodelón, E. (2013). Y en el informe nacional del proyecto GAP Bodelón, E.; Igareda, N.; Casas, G. (2012).

${ }^{6}$ La opción del trabajo cualitativo (Vallés, 1997, 2002; Quivy y Van Campenhoudt, 1992) permite obtener información valiosa que no es posible obtener a través de métodos únicamente cuantitativos. Igualmente permite el análisis de nuevos conceptos, que la investigación quizás no había planteado en las hipótesis iniciales. Muchas investigadoras feministas han preferido la investigación cualitativa pues permite a las víctimas de la violencia sexual articular experiencias con sus propias palabras (Campbell et al. 2009; Jordan, 2001, 2004, 2008; Kelly, 2008; Stanko, 1990).La muestra de participantes de los grupos de discusión y las entrevistas en profundidad no ha pretendido ser representativa. Sin embargo, sí que es suficientemente amplia y variada como para poder obtener información suficiente sobre la realidad de la violencia sexual en las universidades.

${ }^{7}$ En el presente artículo se exponen los principales resultados de la parte cualitativa de la investigación. Sólo cuando sea complementario, se utilizarán algunos de los datos obtenidos en la parte cuantitativa de la investigación. El cuestionario on-line se pasó en la UAB en un solo momento, durante el final del 2009 y principios del 2010. Se consiguió autorización para realizar el envío masivo a todos/as los/las estudiantes de las facultades de Derecho, Medicina, Veterinaria, Filosofía y Letras, Traducción e Interpretación y Ciencias (las estudiantes de todas estas facultades eran 7.840 y representaban el 64,06 \% del total del alumnado de estas facultades). Completaron satisfactoriamente el cuestionario 789 alumnas. El cuestionario on-line se pasó en una segunda fase en las otras cuatro universidades españolas. El total de estudiantes de las cuatro universidades mencionadas anteriormente que completaron de manera satisfactoria el cuestionario on-line fueron de 323 alumnas.
} 
factores de riesgo, consecuencias en sus vidas, cuándo, cómo y a quién comunicaban los episodios vividos, así como las expectativas sobre las autoridades universitarias al respecto. También para averiguar cuál era el conocimiento de los agentes sobre la prevalencia del fenómeno, las responsabilidades que como universidad había al respecto, así su opinión sobre cuáles eran, o deberían de ser los mecanismos de prevención y respuesta ante los episodios de violencia sexual en las universidades

La opción metodológica prioritaria ha sido los grupos de discusión (Krueger y Casey, 2000) porque los grupos focales de discusión facilitan la identificación de información entre los/as participantes y la creación de un discurso colectivo (Morgan, 1997).

El trabajo de campo cualitativo tuvo lugar durante el primer trimestre del 2010, y participaron 32 estudiantes universitarias, en 3 grupos focales de discusión y 4 entrevistas en profundidad ${ }^{8}$. Las estudiantes universitarias fueron invitadas a participar voluntariamente en grupos focales y entrevistas en profundidad a partir del cuestionario anónimo inicial, y también en clases ordinarias de grado, postgrado, máster y doctorado. No era un requisito haber sido víctima de ninguna forma de violencia de género, ni tampoco se las preguntaba directamente sobre esta cuestión, aunque varias de ellas hablaron espontáneamente de su experiencia como víctimas de violencia de género y violencia sexual. También este trabajo de campo cualitativo se completó con entrevistas en profundidad a agentes del ámbito universitario y de fuera de las universidades con competencias, responsabilidades o implicación directa en cuestiones sobre violencia

\footnotetext{
${ }^{8}$ Con el objetivo de entender las citas utilizadas en este informe, hemos codificado los grupos, las
} entrevistas en profundidad y los cuestionarios abiertos online, de la siguiente manera:

\begin{tabular}{|l|l|l|}
\hline \multicolumn{1}{|c|}{ Código } & \multicolumn{1}{c|}{ Tipo de herramienta cualitativa } & \multicolumn{1}{c|}{ Número de personas } \\
\hline FC1 & Grupo Focal & 5 \\
\hline FC2 & Grupo Focal & 5 \\
\hline FC3 & Grupo Focal & 18 \\
\hline II1 & Entrevista en profundidad & 1 \\
\hline I & Entrevista en profundidad & 1 \\
\hline II3 & Entrevista en profundidad & 1 \\
\hline II4 & Entrevista en profundidad & 1 \\
\hline OQ & Cuestionario online & \\
\hline
\end{tabular}


sexual en la universidad. Se entrevistaron a 10 agentes que pertenecían a las estructuras universitarias, a la policía y a la administración. ${ }^{9}$

En España ya se habían llevado a cabo otras investigaciones sobre violencia de género en el ámbito universitario, entre las que podemos destacar: Boch y Ferrer (2000) y Bosch et al. (2006) y Valls (2008a; 2008b). No son investigaciones únicamente limitadas a la violencia de género sexual, sino que son investigaciones que abordan la violencia de género en su totalidad, o estudian alguna forma de violencia de género en particular, como el acoso sexual y el acoso por razón de sexo. Aunque la mayoría de las investigaciones sobre la violencia sexual en el contexto universitario pertenecen al ámbito anglosajón, y en menor medida, a otros países europeos (Bondurant, 2001; Banyard et al. 2005; Gross et al. 2006).

La novedad de la investigación que se muestra a continuación es que los datos se refieren sólo a la violencia de género sexual contra las estudiantes universitarias. No siempre las categorías utilizadas en la investigación pueden relacionarse con categorías legales, ya que en ocasiones pueden encajar total o parcialmente (o en absoluto) en diferentes tipos penales, como por ejemplo bajo los art. 173 del Código Penal (CP) sobre violencia habitual, como violencia física (art. 148.4 CP), como violación (art. 179 CP), abuso sexual (art. 181 y 182 CP), agresión sexual (art. 178 CP), o acoso sexual (art. $182 \mathrm{CP}$ ). Los datos recabados en la investigación se han agrupado en tres grandes formas de violencia de género sexual: el acoso sexual, el stalking y la violencia sexual $^{10}$.

\section{Resultados}

\footnotetext{
${ }^{9}$ Los agentes entrevistados pertenecían 8 de ellos a la universidad (defensor del estudiante, defensora del estudiante de la facultad de derecho, vicedecana para los estudiantes, delegado de la rectora para los estudiantes, directora del observatorio de igualdad, representante de la unidad de asesoramiento psicopedagógico), 1 a la policía y 1 a la administración pública con competencias en materia de seguridad ciudadana.

${ }^{10}$ El tratamiento legal del "stalking” en el ordenamiento jurídico español es incompleto. Si es cierto que a raíz de la Ley Orgánica 3/2007 de igualdad real y efectiva se incluye el acoso por razón de sexo, más allá del acoso sexual, pero en cambio, no se incluyen otras conductas típicas del "stalking" que en otros países se ha hecho. Tampoco hay una traducción unívoca del termino en castellano, de ahí que en este articulo se utilice el termino original de "stalking", más utilizado en Estados Unidos, Australia y Canadá. Vid al respecto Villacampa, 2009.
} 
Las estudiantes universitarias participantes en este estudio tendían a identificar como violencia sexual únicamente las formas más graves de violencia sexual, y no tanto, otras formas de violencia, también de naturaleza sexual como el abuso sexual o el acoso sexual.

De las tres grandes formas de violencia de género (violencia sexual, acoso sexual y por razón de sexo, stalking) estudiadas en esta investigación, la forma más frecuente es el acoso sexual y por razón de sexo (21\% en el caso de la UAB y $24,8 \%$ en el resto de las universidades españolas de las estudiantes encuestadas manifiesta haberlo sufrido frecuentemente), seguido del hostigamiento (stalking) (14\% en el caso de la UAB y $15 \%$ en el resto de universidades españolas de las estudiantes encuestadas) y finalmente la violencia sexual (5,3 \% en la UAB y $8,2 \%$ en el resto de universidades analizadas).

\subsection{Qué entienden las estudiantes por violencia sexual}

Las mujeres estudiantes identifican la violencia sexual de género como las formas más graves de violencia sexual, las formas que constituyen un delito y reciben mayor cobertura por los medios de comunicación y la opinión pública ${ }^{11}$. Otras formas de violencias sexuales como el acoso son en menor medida mencionadas.

"La primera cosa que viene a tu mente es la violación, y luego mientras vamos hablando hay muchas otras cosas que vienen...” (FG2)

"La primera cosa que me viene a la mente cuando hablo de violencia sexual de género es en abuso sexual o violación, se trata de algo muy físico, obligar a alguien a tener relaciones sexuales contra su voluntad. El acoso es más implícito, más sutil, tal vez no tan físico y más psicológico” (FG2)

Las formas menos graves de violencia sexual aparecen también en las entrevistas y grupos de discusión, pero las estudiantes en muchos casos, no están seguras de si lo que habían sufrido podía constituir o no un delito, reconocieron sus dudas acerca de si determinados casos constituyen o no formas de violencia sexual de

11 Está ampliamente descrito la influencia de los medios de comunicación en la criminalización de determinados comportamientos y en la creación de determinados tipos penales. Vid. por ejemplo Fernández, 2009. 
género. Las dificultades que tienen las estudiantes para identificar qué es violencia sexual también han sido corroboradas en investigaciones similares en el ámbito universitario español (Bosch et al., 2006; Valls, 2008a, 2008b), así como en investigaciones sobre violencia sexual en general (Kelly, 1988).

En este sentido, resulta especialmente difícil identificar las formas de acoso sexual si el incidente viene de alguien en una posición superior y más aún si los hechos son inicialmente positivos y agradables, y sólo más tarde, se perciben como formas de agresión por parte de las estudiantes:

“Quizás esta persona te está diciendo cosas bonitas... creo que he sido víctima de acoso sexual, pero esta persona ha empezado a decirme cosas muy bonitas, y me sentí halagada, y en mi caso, era un profesor que yo admiraba y me gustó como estaba enseñando. Tal vez es un crimen desde el primer momento, pero no te das cuenta hasta el sexto mes, cuando hay un momento en que te das cuenta de que algo va mal... Cuando les estás explicando a tus amigos lo que sucede y de repente te das cuenta de que algo malo está pasando..."(FG2)

A pesar de que muchas estudiantes no reconocen en un primer momento haber sufrido la violencia sexual de género, varias de ellas mencionan algunos hechos de su relación con los compañeros, novios o ex novios como episodios de sus vidas que suponen un ataque a su libertad y su auto-estima. Las amenazas de revelar las conductas sexuales o la coacción a través de la sexualidad están siempre presentes en la violencia sexual (Jordan, 2012)

"Me insultaba constantemente por cualquier cosa que hiciera. Me dijo que no le gustaba que me pusiera el pelo en cola de caballo, la forma en que me río, si veo a mis amigos. Me dijo que mi vida sin él no tendría sentido"(OQ)

"iÉl me amenaza diciendo que va a publicar videos filmados durante las relaciones sexuales que no sabía que existían!" (OQ)

\subsection{Las opiniones sobre los casos de violencia sexual.}

En general, las estudiantes participantes piensan que cualquier mujer puede ser víctima de violencia sexual de género, sólo por ser una mujer, ya que esto constituye un factor de riesgo. Aun así, algunas de ellas creen que las estudiantes universitarias son 
especialmente vulnerables a sufrir violencia sexual, porque ven la universidad como un lugar donde hay relaciones de poder muy fuertes y dominantes ${ }^{12}$.

También aparece un tercer grupo de estudiantes, que afirman que las estudiantes universitarias están más protegidas frente a la violencia de género:

"Las mujeres en la universidad tienen los recursos intelectuales para hacer frente a estas situaciones, más que una mujer con bajo nivel educativo. Creo que las mujeres en la universidad tienen una mayor conciencia de la igualdad de género” (II1)

Sin embargo, los datos existentes, tal como ya se ha mencionado, muestran que los estudios universitarios no son una barrera frente a las formas de violencia de género $^{13}$.

Otro debate en el que se reflejaron los estereotipos sobre las violencias machistas fue la cuestión de si ciertos tipos de mujeres, o estilos de vida puedan influir en la posibilidad de que las mujeres sufran mayor riesgo de ser víctimas de violencia sexual. Al principio de los debates, hubo algunas estudiantes que inmediatamente expresaron que cierto tipo de ropa o incluso la belleza de una mujer constituyen un factor de riesgo:

"La sociedad ha creado ciertos estereotipos para justificar la violencia: si usas faldas cortas, o demasiado escote... esto significa que estás provocando" (II4)

Pero cuando hubo la oportunidad de discutir este punto y reflexionarlo con otras estudiantes, se dieron cuenta de que esto no es cierto, o no debería ser el foco del debate, porque las mujeres y los hombres deben tener los mismos derechos y la misma libertad para actuar, comportarse y vestirse como quieran. De esta manera se identificó que el problema es que hay hombres que agreden, que nuestra sociedad puede tolerarlo

\footnotetext{
${ }^{12}$ A la luz de los datos disponibles en España, no parece que las estudiantes universitarias sean un grupo de población que sufra en mayor medida violencia de género, o violencia sexual en particular. Los datos cuantitativos con los que se cuentan, no miden el mismo fenómeno: Macroencuesta de Violencia de Género 2011 (Ministerio de Sanidad, Servicios Sociales e Igualdad, 2012), o l’Enquesta de Violència Masclista a Catalunya del 2010, del Departamento de Interior del Gobierno de Cataluña (Generalitat de Catalunya, 2011) sobre la población en general. Y Bosch et al, 2000, Valls, 2008a, 2008b, Bodelón et al, 2012, sobre la población universitaria.

${ }^{13}$ Ver por ejemplo las diferencias poco significativas por nivel de estudios en la Macroencuesta de Violencia de Género 2011 (Ministerio de Sanidad, Servicios Sociales e Igualdad, 2012).
} 
y aceptarlo en silencio, y que culpabiliza a las mujeres generando la falsa idea de que la razón de las agresiones son estilos de vida. La forma como las mujeres son educadas y socializadas constituye para muchas de ellas la causa real de las violencias:

"No creo que haya un tipo de mujeres, pero creo que debido a nuestra educación podemos caer fácilmente en este tipo de relaciones. Por ejemplo, las presiones familiares para tener una pareja, para mantener la misma pareja, y por supuesto, la idea del "amor romántico" (II3)

La sexualidad juega un papel clave en la comprensión de por qué estos episodios de violencia sexual de género ocurren, quiénes son las mujeres que los padecen y cómo lo viven. Algunas de las mujeres son objeto de críticas precisamente porque viven una vida sexual muy libre y moderna, y esta manera de vivir su sexualidad es vista como la explicación de por qué se convierten en víctimas de violencia de género sexual:

"He oído a un estudiante de Erasmus diciendo que las chicas españolas son muy "fáciles", y pensé que debe ser algo para estar orgullosos, pero luego, si ocurre que esta chica que vive su sexualidad como ella quería y libremente se convierte en víctima de algún ataque y ella lo hace público, los siguientes comentarios llegan: esto te sucede, porque no te comportas como deberías comportarte, y si escucho eso, seguro que me siento culpable" (II4).

\subsection{El contexto de la violencia sexual en las universidades.}

Las mujeres reconocen que la vida universitaria en sí misma constituye un nuevo contexto, en el que se desarrollan formas específicas de violencia sexual. La vida universitaria supone para muchas mujeres jóvenes profundos cambios: vivir fuera de la casa de sus padres, tener libertad para entrar y salir, tener "atípicos" horarios, etc. Estos cambios implican transformaciones en la forma como estas mujeres viven y posibilitan que algunas de ellas puedan romper con los estereotipos de género esperados por la sociedad (Igareda, 2011).

Sin embargo, el nuevo contexto de autonomía que supone la vida universitaria no siempre facilita identificar mejor las situaciones de violencias machistas. La dificultad de reconocerse a sí mismas en situaciones de violencia de género es 
frecuente ${ }^{14}$; a la dificultad que tiene identificar el fenómeno se añaden tópicos como el de que ese tipo de situaciones no se dan entre mujeres universitarias, o como que su condición de universitarias debería haberles posibilitado darse cuenta mucho antes de lo que estaba pasando. Todo ello puede contribuir a generar sentimientos de culpabilidad:

"Cuando estás en la rueda no te das cuenta de cuando llegaste ni de cómo llegaste, porque si eres lo suficientemente fuerte, no entras en esos círculos. Se aprende de estas cosas, y ahora sé lo que habría hecho” (FG2)

"Me siento cabreada porque no vi ciertas señales, porque ese hombre me estaba dando señales claras, que tenía su habitación con las paredes totalmente cubiertas con fotos mías. Pensé que era gracioso y lo tomé como algo normal y totalmente ubicado en el modelo de amor romántico" (II3)

\subsection{Medidas de prevención y autodefensa $^{15}$ de las estudiantes} universitarias

En general, las estudiantes adoptan como estrategia de prevención de las agresiones en los campus ciertos hábitos regulares, y tienen muy en cuenta si se mueven durante el día o por la noche (Igareda, 2012). Evitan ciertos lugares oscuros o aislados de la universidad, o el salir de la biblioteca muy tarde o acudir a la facultad durante los días festivos.

"La biblioteca de la Facultad de Humanidades... Yo no podía salir si estaba sola. Yo estudiaba allí hasta tarde, y si la cierran a las 21:00, trataba de dejarla 10 minutos antes con alguien " (FG1)

"i i iRealmente tengo miedo de la estación de tren en la noche!!!" (FG2)

De la investigación paralela cuantitativa se desprende que las estudiantes universitarias en general se sienten menos seguras en el espacio al aire libre de la

\footnotetext{
${ }^{14}$ Esta dificultad no sólo se desprende de nuestras entrevistas, sino también de otros estudios con mujeres que han sufrido violencias machistas: Bosch et al., 2006; Valls, 2008a, 2008b; Kelly, 1988; Romito, 2007; Creazzo, 2008.

${ }^{15}$ Algunas autoras (Bodelón y Querol, 1999) critican que cuando se introduce la perspectiva de género en el concepto de seguridad, se convierte en miedo en vez de inseguridad, y por lo tanto, una vez más a las mujeres se les atribuye el concepto negativo, asociado a la irracionalidad.
} 
universidad, seguido del parking y el transporte público (Bodelón et al. 2012). Se sienten en cambio más seguras en los edificios universitarios, sean aulas, bibliotecas o cafeterías. Todos estos datos son similares en todas las universidades analizadas, a pesar de que el tipo, localización y tamaño de las universidades participantes era diferente ${ }^{16}$.

También se debe señalar la adopción de hábitos claros de defensa personal para defenderse de un hipotético caso de agresión por parte de algún desconocido. Ellas piensan que son hábitos individuales y extraños, a veces incluso se sienten ridículas al compartirlos con el resto de las miembras del grupo de focal, pero luego se dan cuenta de que casi todas ellas regularmente aplican todo tipo de hábitos de prevención. Estas conductas preventivas se llevan a cabo no sólo en la universidad, sino en el resto de los espacios públicos. Sin embargo, es llamativo que dichas alertas o hábitos preventivos no suelen activarse en el espacio privado, donde ocurren la gran parte de las agresiones.

"Llevo un spray... Confío en que el spray y no en la auto-defensa... Lo compré en Andorra, porque aquí es ilegal, es irritante ..." (FG2)

"Yo no me pongo tacones altos si sé que voy a volver a casa sola, porque no puedo correr tan rápido como con zapatos planos" (FG3)

Estos hábitos han sido aprendidos desde que eran niñas, algunas de ellas directamente recuerdan a sus padres enseñándolas a cuidar de sí mismas. Muchas de ellas los han desarrollado después de escuchar una noticia impactante en los medios de comunicación, o siguiendo los consejos o las malas experiencias de amigas y familiares.

"Ellos te educan en el miedo y la culpa: ten cuidado si te pones minifalda y escote, porque si estás provocando..." (FG3)

Existe una contradicción entre los hábitos regulares de defensa personal y prevención de un ataque sexual o físico ante un agresor desconocido, que como se ha visto, tienen la mayoría de estudiantes universitarias, y el reconocimiento, que ellas mismas hacen, de que son más frecuentes en sus vidas los episodios de violencia de

\footnotetext{
${ }^{16}$ Las universidades participantes están localizadas en puntos bien diferentes de la geografía española. Hay desde universidades pequeñas en número de alumnos/as hasta de las más grandes. También hay universidades localizadas en el núcleo urbano, y otras, como la UAB que están en un campus universitario a las afueras de la ciudad.
} 
género, sea sexual o no, que vienen de hombres conocidos ${ }^{17}$. Estos datos también vienen corroborados por las investigación cuantitativa realizada (Bodelón et al. 2012), así como los otros estudios llevados a cabo en universidades españolas (Bosch y Ferrer, 2000; Bosch et al, 2006; Valls, 2008a, 2008b).

\subsection{La experiencia de la violencia sexual en la vida de las estudiantes y su}

\section{impacto.}

Uno de los impactos más importantes que tiene la violencia sexual es que las mujeres cambian sus hábitos de vida, se sienten más inseguras, su autoestima es dañada, y se sienten culpables de lo que pasó o lo que pueda pasar $^{18}$.

Así, por ejemplo, si el acoso sexual es realizado frecuentemente por compañeros y amigos, como las estadísticas muestran, la víctima no siempre cuenta con la comprensión y ayuda de su entorno más cercano. La sociedad y especialmente su círculo de amigos no siempre entienden que esta manera "normal" de ligar pueda constituir una forma de acoso sexual u hostigamiento para la estudiante. Si la estudiante quiere poner fin a estas conductas, amenaza con hacerlo público y/o denunciar, puede llegar a ser aislada por su propio círculo de compañeros/as y amigos/as, y llegar a ser considerada una persona conflictiva (Igareda, 2011).

"Cuando el acoso sexual o el abuso se da entre compañeros de clase, esto puede provocar aislamiento y rechazo, muchas veces te dicen - ah, tu eres muy dura con este chico pero él es tan agradable... - y en vez de eso tu continúas siendo desagradable con él, y esto explica que seas rechazada por tus propios amigos"(FG1)

\footnotetext{
${ }^{17}$ Las violencias machistas, en general, y las violencias sexuales, en particular, son más difíciles de identificar en el ámbito privado y los mecanismos de prevención también son mucho menores. A las mujeres se nos ha enseñado que el peligro viene de afuera, de los extraños, y por lo general, no imaginamos que la persona que nos gusta o que queremos, pueda ser la persona agresora. Lo paradójico es que la mayoría de las agresiones contra las mujeres ocurren en el espacio privado, que debería ser el espacio de seguridad por excelencia.

${ }^{18}$ En la investigación cuantitativa se vio que en el caso de stalking, el impacto más importante en las víctimas son trastornos emocionales asociados al episodio vivido, sentimientos de tristeza y depresión (38\% de los casos en la UAB) o trastornos de ansiedad o miedo grave (20,4\% de los casos en otras universidades). También es el caso de la violencia sexual, donde en mayor número expresan sentir baja autoestima y humillación (42,9 \% de los casos en la UAB) y su rendimiento académico se ve afectado (el $50 \%$ de los casos en otras universidades).
} 
"Y esto tiene un impacto social, porque se lo conté a mis amigos más cercanos, pero también hablé con un segundo círculo de amigos, pero yo no quería que ellos le transmitieran cierta información a él (el agresor) y hubo algunas personas que no lo entendieron, y me dijeron que era exagerada, iy éstos eran mis amigos!” (II3)

Cualquier tipo de violencia sexual de género es experimentado por muchas mujeres como el peor ataque a la integridad moral y a su dignidad. Las estudiantes expresaron que la forma en que han sido educadas desde que eran niñas, ha incluido el mensaje de que lo peor que les puede pasar es ser violadas o abusadas sexualmente. Su sexualidad es para muchas la cosa más preciada y cualquier ataque a ésta constituye el peor ataque a su dignidad como mujeres.

Otra consecuencia es la culpa que sienten, y la sociedad construye sobre las mujeres agredidas con ideas como la de que una mujer agredida ha hecho algo malo:

"La culpa está conectada con la forma en la que somos socializadas... Sobre todo en mujeres y en las cosas con connotaciones sexuales, y siempre cuando tu honor ha sido manchado, tu eres culpable porque pobres chicos, no pueden soportarlo..." (II4)

Las diferentes formas de violencia sexual también producen consecuencias psicológicas: miedo a salir de sus casas, el pánico a ser secuestradas, fobia a cualquier discusión violenta con la gente que quieren:

"Tuve que quedarme encerrada con mis padres durante todo un mes porque que él estuvo durante muchos días de pie delante de casa de mis padres..." (II3)

"He desarrollado la ansiedad, la depresión... Por otra parte todas las cosas que me dijo no quedan olvidadas, me afectó la forma en que viví mi sexualidad, me tomé tiempo para recuperar mi vida sexual, para hablar de ello... "(II3)

Y por último, los episodios de violencia sexual producen una merma en la libertad de estas mujeres y en su autonomía para vivir como adultas ${ }^{19}$.

"Disminuyó mucho mi movilidad y autonomía, y yo soy una persona muy autónoma y

\footnotetext{
${ }^{19}$ Una de las consecuencias más importantes del acoso sexual constatado en la investigación cuantitativa es que las víctimas tienden a evitar ciertos lugares y situaciones (el 31,\% de los caso en la UAB).
} 
no me gusta pedir que me acompañen a ir a ningún lado, pero cuando esto sucedió, no me gustaba ir a los sitios sola. Y sí, todavía evito ir a ciertos lugares..." (II3)

\subsection{Las dificultades y obstáculos para denunciar las agresiones.}

La opacidad que rodea el tema de las violencias sexuales en las universidades es producto de diversos elementos, tal como hemos visto, pero uno esencial es la dificultad de comunicar y/o denunciar la agresión sufrida. Las razones de ello son variadas.

En primer lugar, las estudiantes manifiestan su miedo a denunciar por la vergüenza que produce hacer público cualquier ataque relacionado con su sexualidad. Históricamente, el padecimiento de una agresión sexual fue un elemento utilizado contra las propias víctimas para cuestionar su sexualidad, su tipo de vida o sencillamente la veracidad de sus palabras. La visibilización de la agresión se convierte así en un hecho complejo en sí mismo y más cuando incluso pueden aparecer amenazas del agresor. Así, por ejemplo, el agresor amenaza a la estudiante con hacer público todo tipo de detalles íntimos sobre su vida sexual si se hace público el ataque.

"Una de sus amenazas era que había fotos mías teniendo relaciones sexuales con él, y mi padre es un político en $X, y$ una de sus amenazas era que él iba a publicar estas fotos y distribuirlas en el trabajo de mi padre. Por lo tanto la opción de contárselo a mi familia era imposible, yo nunca les hablé de estas fotos..." (II3)

Otras veces, el simple hecho de hablar de la agresión en público, es muy vergonzoso de cara a dar el paso de denunciar:

"La humillación que sentí en ese momento, la vergüenza al decírselo a mis padres, a mis amigos.... Yo estaba tan avergonzada al contar que mi profesor de conducir me estaba tocando y me decía cosas sucias... porque hablar de cosas sexuales es muy difícil, y yo tenía miedo de que no me creyeran, de que la gente pudiera pensar que yo estaba exagerando. Tenía miedo del caos que iba a producir en la escuela de conducir... así que lo sufrí sin contárselo a nadie, y evité ponerme faldas y escotes.... $Y$ esperé a aprobar el examen tan pronto como fuera posible para salir de la escuela de conducir" (II2)

"Fue más complicado decírselo a mi familia... por otra parte yo no vivía con ellos, yo no iba a llamar y decir: - Mira lo que me pasó..."-(II.4) 
En segundo lugar, las estudiantes no tienen una información clara sobre qué conductas son denunciables en sede jurídica. La falta de información también se debe a la falta de conocimiento sobre si el incidente constituye un delito o no, especialmente si es cometido por alguien conocido, o si el incidente no constituye un grave ataque físico, más fácil de probar.

"Tal vez el episodio de la escuela de conducir.... Nunca pensé que este hombre pudiera ir a la cárcel por algo así, pensé que podría ser despedido si yo lo denunciara" (II2)

Cuando no informan o denuncian, tratan de justificarlo basándose en que consideran los incidentes como algo no tan importante, no tan grave, sobre todo porque el incidente no fue físico o no dejó secuelas físicas:

"Yo no le di importancia, ellos estaban borrachos y se lo estaban haciendo a todas las chicas que pasaban" (OQ)

Esto también explica por qué en muchos casos no se denuncian. En la investigación cuantitativa las razones más citadas por las estudiantes encuestadas para no comunicar a nadie los episodios de violencia sexual vivida (acoso sexual, stalking y violencia sexual) eran porque que consideraban que era un incidente aislado que ya había finalizado, porque lo único que querían era que aquello terminara y que las dejaran en paz, por miedo a no ser creídas o atendidas adecuadamente. Cuando las estudiantes comunican a alguien lo que ha ocurrido, lo hacen sólo a familiares y amigos, y casi nunca a las autoridades universitarias, y aún menos a la policía (Bodelón et al., 2012).

"Pasaron dos años y medio de acoso psicológico y, cuando estaba a punto de golpearme me fui del lugar, y yo no tenía las agallas para denunciar... Y lo mejor que hice fue cortar todas las comunicaciones, salir y desaparecer, cambiar el teléfono.... Porque cuando estás ahí (en una situación de violencia de género) tú no eres tú misma" (FG2)

"Cuando el incidente se convierte en público, la víctima comienza a auto-culparse cómo he permitido que algo así me pase a mí, por qué no reaccioné antes-, todo este proceso de culpa y vergüenza aparece una vez que lo has hecho público. Es 
especialmente difícil dar el paso de denunciar si sabes que tendrás que enfrentarse con esta mirada externa y que busca culpar desde fuera” (II4)

En tercer lugar, las estudiantes no confían en las instituciones, así por ejemplo, conocen las nuevas formas de violencia sexual de género relacionadas con las drogas, pero no las denuncian porque temen no ser tomadas en serio:

"Ahora hay drogas sintéticas que te hacen perder la memoria por completo, o sólo tienes algún recuerdo después. Las chicas que despiertan en sus camas, en sus casas abiertas con sus llaves, pero no saben lo que ha sucedido, y seguramente han sido agredidas sexualmente. Esta es una circunstancia muy complicada, porque si van al hospital, le dirán: esta chica es una fiestera y no quiere admitir que ha tomado drogas y se lo pasó muy bien... Esto está sucediendo ahora, así que otra medida de seguridad: no dejes tu vaso solo" (FG3)

Ninguna de las estudiantes entrevistadas ha denunciado a la policía ${ }^{20}$. No confían en que esto pueda cambiar las cosas o se les trasmite el mensaje de que la denuncia no va a protegerlas ni a reparar el daño, aunque en realidad desconocen qué mecanismos de protección activa la denuncia o qué posibilidades de reparación del daño existen. ${ }^{21}$.

"Le pedí ayuda a un amigo juez de mi familia, y él me dijo que como el agresor se preparaba para entrar en las fuerzas policiales, si hago una denuncia que suponga poner fin a su futura carrera de policía, esto constituía una razón contra mí. Yo tenía mucho miedo a denunciar. Además, la pena máxima a imponer era una orden de alejamiento de 6 meses, y si se rompe una orden de alejamiento no es para venir a conversar conmigo, así que después de 6 meses me fui a estudiar al extranjero” (II3)

Algunas de ellas piensan que no era necesario, y que fue suficiente con informar a los servicios de seguridad de la universidad, por ejemplo, porque se trató de un incidente que tuvo lugar en el campus, y por lo tanto, entendieron que era una competencia sólo de la universidad.

\footnotetext{
${ }^{20}$ Esto igualmente aparece en estudios sobre violencia sexual en la sociedad en general (Fischer et al. 2003, Jordan, 2001, 2004; Stern, 2010).

21 También esto es común en otras investigaciones sobre violencia sexual en general (Daly y Mahler (1998).
} 
Como en otros casos de violencia de género, el miedo a no obtener una respuesta satisfactoria, a la impunidad y la ausencia de respuesta institucional es una de las causas que apuntan los estudios cualitativos sobre agresiones sexuales (Sortzen, 2011). Estos elementos son también los indicados en el último informe europeo sobre las violaciones en Europa realizado por el European Women's Lobby, que afirma asimismo que aunque el reto es aumentar el número de denuncias, no se debe olvidar que se deben facilitar sistemas de protección y reparación del daño a las víctimas (European Women's Lobby, 2013).

La mayoría de las estudiantes que han vivido algún tipo de violencia sexual de género, lamentan no haberlo hecho público lo suficiente. A veces tenían demasiado miedo, no querían pasar por todo el examen público. Sin embargo, admiten que deberían haberlo hecho más público, porque esta publicidad habría resuelto el problema, poniendo fin a más incidentes futuros, evitando nuevos ataques a otras estudiantes y tal vez, ayudando a otras estudiantes a denunciar los mismos hechos.

"Creo que estos casos deben hacerse públicos, para ayudar a otras personas, por ejemplo, a denunciar. Tal vez no eres la única chica que lo sufre...” (II2)

"Ahora me siento mal porque yo no hice nada, y probablemente este hombre se lo hizo a otras chicas, y si nadie dijo nada, probablemente este hombre ha seguido haciéndolo y se ha retirado sin problemas" (II2)

Los agentes entrevistados en general desconocen la magnitud e incluso la existencia o no de la violencia sexual en la universidad. A excepción de algunos recursos que están en contacto directo con la población universitaria, porque desarrollan labores de asesoramiento psicopedagógico, y desempeñan algunas funciones de prevención y formación, la mayoría de ellos creen que la violencia sexual debe ser un fenómeno muy raro y aislado, y se apoyan en la ausencia de denuncias en la historia más reciente de la universidad.

"En un año y medio como Síndic, no ha habido ningún incidente de violencia sexual (...) creo que la $U A B$ es una universidad tranquila y no sé si la gente no denuncia o reclama porque tienen miedo o vergüenza” (Síndic - Defensor de la Universidad). 
Los agentes entrevistados también manifiestan cómo a veces la mal entendida autonomía universitaria, dificulta la denuncia de los delitos (como es el caso de la violencia sexual) ocurridos en la universidad, y también previene a las autoridades universitarias de actuar frente a estos episodios.

Únicamente el jefe de la policía local entrevistado tenía una idea clara sobre cuál debería ser el procedimiento a seguir en un episodio de violencia sexual en la universidad $^{22}$. Esta persona distinguía los actos de los estudiantes que pueden llegar a ser una infracción administrativa, y por lo tanto, se aplicaría el Régimen Disciplinario ${ }^{23}$ bajo la autoridad del rector, y los actos constitutivos de delito, como puede ser las diferentes formas de violencia sexual, donde debería aplicarse el Código Penal y la Ley Integral de Violencia de Género.

“Esto está bajo la autoridad del rector/a. El único caso en el que podemos intervenir sin la autorización del rector/a es en el caso de delitos flagrantes (...) si constituye una infracción administrativa, el rector/a es la autoridad competente, pero si se trata de un delito penal, ya no, pero si no somos informados, no podemos hacer nada" (Jefe de la policía local).

"No deberíamos tener miedo a abordar la cuestión de seguridad pública dentro de las universidades...y aún menos en el caso de nuestra universidad que es un campus....no tiene nada que ver con la autonomía universitaria (..) sino esto implicaría que se podrían dar delitos con total impunidad, esto me preocupa muchísimo” (El delegado del rector de la UAB para los estudiantes)

Igualmente la mayoría de los agentes entrevistados consideran que sería necesario un protocolo de actuación de la universidad en casos de violencia de género, que incluiría no sólo orientación y asesoramiento, sino también derivación a recursos

\footnotetext{
${ }^{22}$ A pesar de que son la policía nacional o la policía autonómica quienes tienen la competencia principal sobre los casos de violencia de género, la policía local juega un papel fundamental en aquellos territorios en los que existe un protocolo de actuación integral en los casos de violencia de género, donde se coordinan todos las posibles instancias para asegurar una respuesta integral a las mujeres víctimas de violencia de género (ayuntamiento, servicios sociales, servicios de salud, casa de acogida, policía local, policía autonómica o nacional, etc.)

${ }^{23}$ El Régimen Disciplinario de los estudiantes universitarios es una norma aprobada en 1954, durante el régimen político franquista pero todavía está vigente. Incluye diferentes sanciones administrativas para aquellos estudiantes cuyos actos puedan constituir una amenaza a la vida universitaria y/o a para otros estudiantes. Aunque es sumamente criticada por las asociaciones y sindicatos de estudiantes, se aplica en raras ocasiones, y las personas entrevistadas no recordaban que se hubiera aplicado en casos de violencia de género cometidos en el territorio de la universidad.
} 
especializados para las posibles víctimas. Este protocolo serviría tanto para que toda la comunidad universitaria supiera qué hacer ante un episodio de violencia de género en la universidad (estudiantes, profesores y autoridades responsables), e igualmente, para cumplir con la obligación de dar el mayor apoyo y atención integral a la víctima dentro de las responsabilidades de la universidad.

"Yo creo que sería muy útil....para todo el mundo, porque es muy fácil que el estudiante no sepa qué hacer...y su único dilema sea si tiene que ir o no a la policía, y ella ni siquiera piense que la universidad pueda ayudarla porque considere que esto no es una cuestión académica...(...) pero si hay un protocolo, puedes decirla - eh, mira, si te pasa esto, tu puedes hacer esto y aquello - y sería muy útil también para el profesorado porque también te puede llegar a tí en un momento determinado" (La síndicaDefensora de la facultad de Derecho).

"Aparte de eso, sería necesario contar con alguien especializado en la universidad, con un conocimiento mínimo sobre la materia, suficiente para identificar situaciones de violencia de género (...) el vínculo podría ser una persona, o una línea telefónica, como en otros sitios, pero aquí en el campus de la $U A B$, algún sitio donde puedas llamar o acudir para hablar...donde puedan informarte en qué debes hacer y con qué recursos cuentas para ayudarte” (Unidad de asesoramiento psicopedagógico)

A la luz de las declaraciones de los agentes entrevistados, no es de extrañar que las estudiantes sean reticentes a comunicar a las autoridades universitarias, y que no piensen en denunciar. La gran mayoría de dichas autoridades (a excepción de las autoridades policiales de fuera del campus), prácticamente desconocen cuál debería ser el procedimiento a seguir si alguna estudiante comunicara un episodio de violencia sexual en la universidad.

"Cuando aquel profesor me comentó lo que estaba ocurriendo (en relación a un estudiante que estaba siendo acosado sexualmente por alguien desconocido) me dije: no tengo ni idea qué hacer...y bueno fui a la Secretaria General (cargo ocupado por una profesora de Derecho) porque tenemos relación de compañeras, sino... bueno, supongo que hubiera contactado a los servicios jurídicos ...sino no hubiera sabido qué hacer...porque estas cosas están fuera de nuestras funciones normales como profesores, normalmente vinculadas a cuestiones académicas: quejas sobre un examen, sobre el expediente académico, etc. Esta es otra dimensión que no hemos asumido en absoluto" (Síndica - Defensora de la Facultad de Derecho) 


\section{Conclusiones}

La combinación de la metodología cuantitativa y cualitativa de esta investigación (aunque en este artículo se haya presentado esencialmente los resultados cualitativos) nos permite extraer algunas conclusiones sobre las características de la violencia sexual en la universidad, así como las dificultades para identificar este fenómeno y denunciarlo por parte de las estudiantes universitarias. Asimismo nos ofrece la oportunidad de plantear un debate sobre el conocimiento y actual respuesta de las autoridades universitarias al respecto, y cuál debería ser el nivel de responsabilidad ideal.

Una reflexión significativa es pensar por qué la educación superior tiene como efecto una mayor información de las estudiantes. Las estudiantes tienen dificultades en identificar lo que sufren como violencia sexual: generalmente han sido socializadas para prevenir y defenderse frente a un eventual agresor extraño, cuando en la mayoría de los casos, el agresor es alguien conocido, o incluso alguien con el que se mantiene o ha mantenido una relación afectiva. Paradójicamente han sido socializadas para defenderse ante un eventual ataque de un agresor que no conocen, para los que han desarrollado mecanismos de autodefensa y hábitos preventivos en su vida diaria. No informan a las autoridades universitarias, y aun menos denuncian, por la vergüenza, el estigma, el miedo a no ser creídas, el temor a sentirse aisladas, o a que la respuesta policial y/o legal no sirva de nada. En todo caso, si lo comunican a alguien es generalmente a sus amigos/as, a su entorno más íntimo, ante el tabú y la vergüenza que se asocia a todo aquello relacionado con la sexualidad.

Obviamente los resultados de la investigación cualitativa presentada en este artículo fueron realizados sólo en una universidad pública, pero muchas de sus conclusiones pueden ser extrapolables a la población universitaria femenina española, ya que como se ha visto, las características físicas de las universidades no tienen tanto peso en la prevalencia y vivencia de las violencias sexuales en las estudiantes universitarias como pudiera pensarse a priori.

A pesar de la ausencia de datos y denuncias sobre las diferentes formas de violencia sexual en las universidades (tal y como ha evidenciado también la presente investigación), no por ello deja de existir, y se trata de una grave violación de los 
derechos fundamentales de las estudiantes sobre la que es necesario actuar, tanto de manera preventiva como en la respuesta integral. Sus fuertes consecuencias en la salud física, mental y en la libertad y autonomía de estas mujeres requieren una implicación del ámbito universitario, un compromiso que haga también en este tema a las universidades un lugar de excelencia presidido por la ausencia de violencias machistas.

Son pocas las universidades en el contexto estatal y europeo que cuentan con medidas específicas de prevención y de respuesta integral frente a la violencia de género, y la violencia sexual en particular. Las autoridades universitarias salvo excepciones no tienen un conocimiento muy profundo del tema, y en general consideran que se trata de episodios aislados, que se escapan de sus competencias ordinarias.

\section{Agradecimientos}

Nos gustaría agradecer la participación generosa de todas las estudiantes universitarias que participaron en los cuestionarios on-line, los grupos de discusión y que se prestaron voluntariamente a las entrevistas en profundidad, para hablar muchas veces de experiencias traumáticas y sumamente íntimas de sus vidas. También a todos los agentes que nos brindaron su tiempo desde la responsabilidad y la seriedad en el ejercicio de sus cargos. También a las sugerencias y comentarios de los evaluadores de la REIC que han enriquecido el presente artículo.

\section{Financiación}

Esta investigación ha sido realizada en el marco del Proyecto GAP "Gender-based violence, stalking and fear of crime” (2008-2011) financiado por la Comisión Europea bajo el programa "Prevention of and Fight Against Crime Programme". Coordinado por la Universidad de Bochum (Alemania), participaron la Universidad de Keele (Inglaterra), Bolonia (Italia), Cracovia (Polonia) y la Universitat Autònoma de Barcelona. 


\section{Referencias}

Banyard, V.L., Plante, E.G., Cohn, E.S., Moorhead, C; Ward, S. y Walsh, W. (2005). Revisiting unwanted sexual experiences on campus. A 12 year follow-up. Violence Against Women, 11(4): 426-446.

Bodelón, E. y Querol, E. (1999). Differenza di genere e politiche di sicurezza nelle città europee, Bologna : Regione Emilia-Romagna.

Bodelón, E.; Igareda, N. y Casas, G. (2012). Violencia sexual, acoso y miedo al delito. Informe español, Bochum: Universidad de Bochum. Disponible en: http://vmrz0183.vm.ruhrunibochum.de/gendercrime/pdf/gendercrime_country_r eport_spain_spanish.pdf

Bosch, E. y Ferrer, V. (2000). Assetjament i violència de gènere, Palma: Documenta Balear.

Bosch, E., Ramis, C., Ferrer V. y Navarro, C. (2006). Las violencias contra las mujeres en la pareja: creencias y actitudes en estudiantes universitarios/as. Psicothema, 18 (3), 359-366.

Bondurant, B. (2001). University's women acknowledge of rape. Violence Against Women, 7(3): 294-314.

Bourke, J. (2009). Los violadores. Historia del estupro de 1860 a nuestros días. Barcelona: Ed. Crítica.

Cantera, L. (1999). Te pego porque te quiero. La violencia en la pareja. Barcelona: Publicaciones Universitat Autònoma Barcelona..

Campbell, R., Adams, A., Wasco, S., Courtney, A. y Self, T. (2009). Training interviewers for research on sexual violence: a qualitative study of rape survivors' recommendations for interview practice. Violence Against Women, 15(5): 595-617.

Creazzo, G (ed). (2008) Scegliere la libertà: affrontare la violenza. Milano: Franco Angeli.

Daly, K. y Mahler, L. (ed.) (1998). Criminology at the crossroads. Feminist Readings in Crime and Justice. New York: Oxford University Press.

Dobash, E. y Dobash, R. (1992). Women, violence and Social Change. London: Routlegde.

European Union Agency for Fundamental Rights, (2014), Violence against women:

an EU-wide survey: Main results. Luxembourg: Publications Office of the European Union. 
European Women's Lobby, (2013). EWL Barometer on rape in the EU, Bruselas, European Women's Lobby, disponible en: http://www.womenlobby.org/spip.php?article5119\&lang=en.

Fernandez, M. (2009). Dones i seguretat. Revista Catalana de Seguretat Pública. 20: 85-97.

Fisher, B., Diagle, L., Cullen., F. y Turner, M. (2003). Reporting sexual victimization to the police and others: Results from a national-level study of college women. Criminal Justice and Behaviour. 30 (1): 6-38.

Generalitat de Catalunya (2010). Enquesta de violència masclista a Catalunya, disponible

en http://www20.gencat.cat/docs/interior/Home/MS\%20\%20Pla\%20seguretat\%20i \%20atencio\%20victimes/03\%20Materials\%20i\%20dades\%20sobre\%20violènci a\%20masclista\%20i\%20domèstica/Documentacio\%20sobre\%20violencia\%20m asclista\%20i\%20domestica\%20per\%20a\%20professionals/Enquesta\%20de\%20 violencia\%20masclista/PresentacióEVMC.pdf.

Gil J.M. (2007). Los diferentes rostros de la violencia de género. Madrid: Dykinson,Gregory, J. y Lees, S. (1999). Policing sexual Assault, London: Routledge.

Gross, A.M.; Winslett, A.; Roberts, M. y Gohm, C.L. (2006). An examination of sexual violence against college women. Violence against women, 12 (3):288-300.

Hanmer, J.; Radford, J. y Stanko, E. (1989). Women, policing and male violence, London y New York: Routledge.

Igareda, N. (2011). La voz de la estudiantes. Las violencias sexuales en el ámbito universitario. En Rodriguez R. y Bodelón, E. (coords.). Las violencias machistas contra las mujeres. Barcelona: Bellaterra, 29-40.

Igareda, N. y Bodelón, E. (2013). Las violencias sexuales y el acoso sexual en el ámbito universitario español. Rivista di Criminologia, Vittimologia e Sicurezza. 7(2): 65-79.

Jordan, J. (2001). Worlds apart? Women, rape and the reporting process. British Journal of Criminology 41 (4): 679-706.

Jordan, J. (2004). The Word of a Woman? Police, Rape and Belief. Houndmills, Basingstoke: Palgrave Macmillan.

Jordan, J. (2008). Serial Survivors: Women's Narratives of Surviving Rape. Sydney: The Federation Press.

Jordan, J. (2012). Silencing rape, silencing women. En Brown, J. M. y Waklate, S. L. Handbook on sexual Violence, London: Routledge. 
Kelly, L. (1988). Surviving Sexual Violence.Cambridge: Polity Press.

Krueger, R. A. y Casey, M.A. (2000). Answering Questions about the Quality of Focus Group Research. En R.A Krueger,. y M. A. Casey (eds).Focus Groups: A Practical Guide for Applied Research. Thousand Oaks, CA: Sage.

Lorente, M. (2001). Mi marido me pega lo normal. Agresión a la mujer: realidades y mitos. Barcelona: Editorial Ares y Mares.

Morgan, D. (1997). Focus Groups as Qualitative Research, Thousand Oaks, London, New Delhi: Sage.

Ministerio de Sanidad, Servicios Sociales e Igualdad (2012). Macroencuesta de Violencia de Género, 2011, disponible en http://www.observatorioviolencia.org/upload_images/File/DOC1329745747_ma croencuesta2011_principales_resultados-1.pdf.

Quivy, R. y Van Campenhoundt, L. (1992). Manual de investigación en ciencias sociales. Mexico, D.F.: Limusa Noriega.

Romito, P. (2007), Un silencio ensordecedor. La violencia ocultada contra mujeres y niños. Madrid: Montesinos.

Sortzen, C. (2011). Agresiones sexuales. Cómo se viven, cómo se entienden y cómo se atienden. Gasteiz: Servicio de Publicaciones del Gobierno Vasco.

Stanko, E.(1990). Everyday violence. How women and men experience sexual and physical danger. London: Pandora.

Stern, V. (2010). Stern Review of Rape Reporting in England and Wales. London: Home Office.

Vallés, M. S. (1997). Técnicas cualitativas de investigación social. Reflexión metodológica y práctica profesional. Madrid: Síntesis.

Vallés, M. (2002). Entrevistas cualitativas. Madrid: Centro de Investigaciones Sociológicas.

Valls, R.; Oliver, E.; Sánchez Aroca, M.; Ruiz Eugenio, L.; Melgar, P.; (2007) Violencia de género también en las universidades? Investigaciones al respecto. Revista de Investigación Educativa, 25 (1): 219-231.

Valls, M.R (Dir.) (2008a). Violències de gènere en l'àmbit universitari? Realitats, formes i superació. Informe resum dels resultats de la recerca. Barcelona: AGAUR.

Valls, M.R. (ed.) (2008b). Violencia de género en las universidades españolas, Madrid: Ministerio de Igualdad. 
Villacampa C. (2009). Stalking y derecho penal. Relevancia jurídico-penal de una nueva forma de acoso. Madrid: Iustel.

World Health Organisation, (2005). Multi country study on women's health and domestic violencen against women. Ginebra: World Health Organization.

Noelia Igareda es profesora acreditada lectora de Filosofía del Derecho, Universidad Autónoma de Barcelona (UAB). Profesora Criminología UAB. Doctora en Derecho. Máster en Políticas de Igualdad de Género: Agentes de Igualdad (UAB). Licenciada en Derecho, especialidad jurídico-económica por la Universidad de Deusto. Investigadora en el grupo de investigación: Antigona. Grupo de Investigación sobre Derecho y sociedad en perspectiva de género, de la UAB. http://antigona.uab.cat/

Encarna Bodelón es Profesora Titular de Filosofia del Derecho, de la Universidad Autónoma de Barcelona. Profesora de Criminología de la UAB. Doctora en Derecho. Postgrado en Sistema Penal y problemas sociales (UB). Master en estudios de género (Utrecht). Directora del grupo de investigación: Antigona. Grupo de Investigación sobre Derecho y sociedad en perspectiva de género, de la UAB. http://antigona.uab.cat/ Co-directora del postgrado Género e Igualdad de la UAB. 